\title{
Intrazerebrale Blutungen
}

\section{Das Spot-Sign in der CT-Angiografie sagt das "Wachstum" vorher}

Demchuk AM, Dowlatshahi D,

Rodriguez-Luna D et al; for the PRE-

DICT/Sunnybrook

ICH CTA study group. Prediction

of haematoma growth and outcome in patients with intracerebral haemorrhage using the CT-angiography spot sign (PREDICT):

a prospective observational study. Lancet Neurol 2012;

11: $307-14$
Fragestellung: Kann das Spot-Sign in der CT-Angiografie voraussagen, ob eine intraparenchymatöse Hirnblutung größer wird?

Hintergrund: Zerebrale Blutungen machen 7-15\% aller Schlaganfälle aus und haben eine schlechte Prognose mit einer Sterblichkeit von bis zu $40 \%$. Die wichtigsten Prädiktoren für einen schlechten Outcome sind die initiale Größe der Blutung, die Bewusstseinslage, der Nachweis von Blut in den Ventrikeln, das Lebensalter und die Größenzunahme der Blutung in den ersten Stunden. Bisher gab es keinen guten Parameter, der die Größenzunahme einer Hirnblutung voraussagen würde. Dies könnte auch erklären, warum die Therapiestudien zu rekombinantem Faktor VII negativ verliefen, da hier Patienten mit und ohne Größenzunahme der Blutung in einer Studie vermischt wurden. Das sogenannte SpotZeichen in den Quellenbildern der CT-Angiografie zeigt Kontrastmittelaustritt in das Hirnparenchym und belegt,

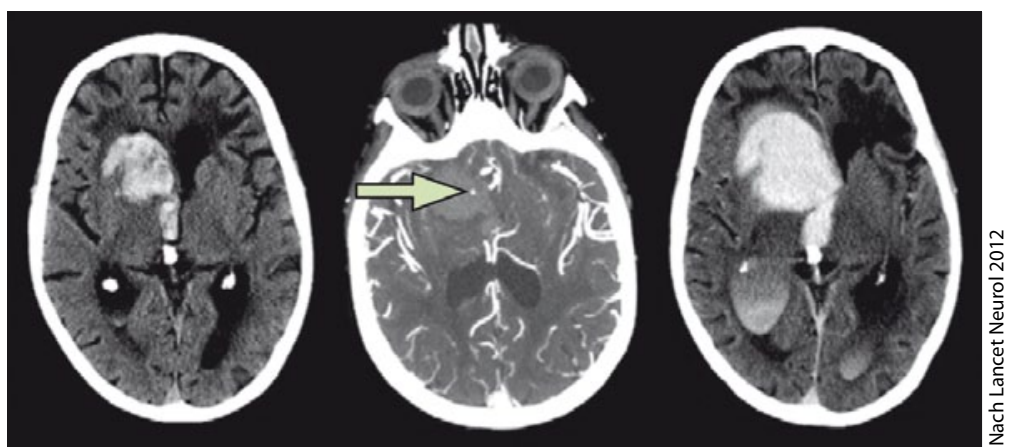

Abbildung 1 Links: Baseline Nicht-Kontrast-CT (Hämatomvolumen 19,6 ml). Mitte: Baseline CT-Angiografie (einzelnes Spot-Sign). Rechts: 24-h-Follow-up Nicht-Kontrast-CT (Hämatomvolumen 110,8 ml)

Kommentar: Diese große multizentrische prospektive Studie belegt eindeutig, dass das Spot-Sign ein guter Prädiktor für die Größenzunahme einer intraparenchymatösen Blutung ist und auch den Outcome bezüglich neurologischer Ausfälle und Mortalität voraussagt. Die Bestimmung ist sehr einfach, weil lediglich eine CT-Angiografie erforderlich ist. Das Spot-Sign wird in Zukunft ein wichtiger Parameter sein, um Patienten in Therapiestudien bezüglich ihrer Prognose zu stratifizieren. Studien mit hämostatischer Therapie sollten daher ganz überwiegend Patienten mit positivem Spot-Zeichen einschließen, da es hier am wahrscheinlichsten ist, einen Unterschied in der Therapie zwischen aktiver Substanz und Placebo zu zeigen.

Hans-Christoph Diener, Essen dass offenbar noch Gefäßwände undicht sind (Abbildung 1). Dieses Spot-Zeichen könnte ein Prädiktor für eine Größenzunahme einer bestehenden Hirnblutung sein.

Patienten und Methodik: Es handelt sich um eine prospektive multizentrische Beobachtungsstudie. Eingeschlossen wurden Patienten mit einer Hirnblutung von weniger als $100 \mathrm{ml}$ Volumen, die sich innerhalb von sechs Stunden nach Beginn der Symptomatik vorstellten. Bei allen Patienten wurden initiale CT durchgeführt und nach dem Spot-Sign in der CT-Angiografie geschaut. Ein Neurologe, der über den klinischen Zustand des Patienten keine Informationen hatte, bestimmte das Volumen der intrazerebralen Blutung mit einer computerisierten Planimetrie. Der primäre Endpunkt der Studie war eine Zunahme der Blutung definiert als eine absolute Volumenzunahme von $6 \mathrm{ml}$ oder mehr oder eine relative Volumenzunahme von $33 \%$ zwischen dem initialen CT und dem nachfolgendem CT.

Ergebnisse: Es wurden 268 Patienten in die Studie aufgenommen. Die mittlere Zeit von Beginn der Symptomatik bis zum initialen CT war 135 Minuten und bis zur Durchführung der CT-Angiografie 159 Minuten. 81 Patienten (30\%), hatten ein positives Spot-Sign. Bei 228 Patienten lagen ein initiales und ein Nachfolge-CT vor. Bei diesen Patienten war keine Operation durchgeführt worden und es handelte sich um überlebende Patienten. Der Median des Blutungsvolumens beim ersten CT betrug 19,9 ml. Die mediane Ausdehnung der Blutung betrug 8,6 ml für Patienten mit positivem Spot-Sign und $0,4 \mathrm{ml}$ für Patienten mit negativem Spot-Sign. Dieser Unterschied war statistisch signifikant. Der klinische Outcome, gemessen mithilfe der modifizierten RankinSkala nach drei Monaten, betrug fünf Punkte bei den Patienten die ein Spot-Sign aufwiesen und drei Punkte bei den Patienten, bei denen kein Spot-Sign nachgewiesen werden konnte. Die Sterblichkeit nach drei Monaten betrug in der ersten Gruppe $43,4 \%$ und in der zweiten Gruppe 19,6\%. Beide Unterschiede bezüglich des Outcomes waren statistisch signifikant.

Schlussfolgerungen: Diese prospektive multizentrische Beobachtungsstudie belegt, dass das Spot-Sign in der CT-Angiografie ein wichtiger Prädiktor für die Größenzunahme einer parenchymatösen Hirnblutung ist. Das Spot-Sign sollte ein wesentliches Kriterium bei der Durchführung von Therapiestudien bei intrazerebralen Blutungen sein. 